\title{
Multiplicity fluctuations in high-energy nuclear collisions as signature of the temperature fluctuations
}

\section{Grzegorz Wilk*}

The Andrzej Sottan Institute for Nuclear Studies, Hoza 69, PL-00681 Warsaw, Poland

E-mail: wilk@fuw.edu.pl

\section{Zbigniew Włodarczyk}

Institute of Physics, Jan Kochanowski University, Świętokrzyska 15, PL-25406 Kielce, Poland

E-mail: zbigniew.wlodarczyk@ujk.kielce.pl

The large number of produced secondaries observed in recent heavy ion experiments (and still larger multiplicities expected to come from the LHC experiments) allows to study different aspects of dynamics of hadronization process on event-by-event basis. Among them the experimentally measured multiplicity fluctuations are especially important as a potential source of information on the strongly interacting system formed in such collisions. Most of studies of such processes use a statistical approach based on the Boltzmann-Gibbs (BG) statistics. However, conditions for the use of BG approach are usually not fully met in such reactions and one encounters some inherent problems arising from the smallness of the collision system and from its rapid evolution (the spatial configuration of the system are far from uniform and global equilibrium is not established). Nevertheless, one can still use simple statistical approach provided it is based on the nonextensive extension of the BG statistics with a new additional nonextensivity parameter $q$. In this extension, usually called Tsallis statistics, parameter $q$ accounts summarily for all dynamical factors resulting in violation of assumptions of BG statistics. It characterizes itself by power-law form of basic distribution (see [T.S. Biró (ed.), Eur. Phys. J. A 40(3)(2009)] for recent review on this subject; for $q \rightarrow 1$ the usual BG approach with the usual exponential distributions is recovered). In this presentation we shortly review the most interesting results of our studies (done using nonextensive statistics) of the recently observed fluctuations of multiplicity in heavy ion collisions.

European Physical Society Europhysics Conference on High Energy Physics, EPS-HEP 2009, July 16 - 222009

Kraków, Poland

\footnotetext{
* Speaker.
} 
Because statistical models are the most useful tools for investigations of different phenomenological aspects of multiparticle production processes, the natural question one can ask is whether they can also accommodate phenomena of fluctuations which are more and more visible in heavy ion collisions [1]. The answer is positive provided such models are based on nonextensive statistics with a new, additional to the temperature $T$, parameter $q$ accounting for such fluctuations, usually understood as fluctuations of the temperature $T$ [2] (among other things, see [3] for the collection of the most recent mini-reviews on different aspects of nonextensive approach).

To demonstrate the usefulness and importance of nonextensive approach when studying multiplicity fluctuations let us remind the following observation (discussed in $[1,4,5]$ ). Whenever in some process $N$ independent particles with energies $\left\{E_{1, \ldots, N, N+1}\right\}$ follow the exponential (Boltzmann) joint probability distribution

$$
g\left(\left\{E_{1, \ldots, N}\right\}\right)=\frac{1}{\lambda^{N}} \exp \left(-\frac{1}{\lambda} \sum_{i=1}^{N} E_{i}\right), \quad \lambda=\langle E\rangle
$$

and satisfy condition $\sum_{i=0}^{N} E_{i} \leq E \leq \sum_{i=0}^{N+1} E_{i}$, then the corresponding multiplicity $N$ has a Poissonian distribution,

$$
P(N)=\frac{\langle N\rangle^{N}}{N !} \exp (-\langle N\rangle), \quad\langle N\rangle=\frac{E}{\lambda} .
$$

However, when Boltmann distribution (1) is replaced by Tsallis distribution [3] of energies,

$$
h\left(\left\{E_{1, \ldots, N}\right\}\right)=C_{N}\left[1-(1-q) \frac{\sum_{i=1}^{N} E_{i}}{\lambda}\right]^{\frac{1}{1-q}+1-N},
$$

then, for energies $E$ satisfying the same condition as before, the corresponding multiplicity distribution is equal to the well-known negative-binomial (NB) distribution,

$$
P(N)=\frac{\Gamma(N+k)}{\Gamma(N+1) \Gamma(k)} \frac{\left(\frac{\langle N\rangle}{k}\right)^{N}}{\left(1+\frac{\langle N\rangle}{k}\right)^{N+k}} \quad \text { with } \quad k=\frac{1}{q-1}, \quad\langle N\rangle=\frac{E}{\lambda},
$$

for which $\operatorname{Var}(N)=\frac{E}{\lambda}\left[1-(1-q) \frac{E}{\lambda}\right]=\langle N\rangle+(q-1)\langle N\rangle^{2}$. Notice that for $q \rightarrow 1$ one has $k \rightarrow \infty$ and $P(N)$ becomes a Poisson distribution whereas for $q \rightarrow 2$ one has $k \rightarrow 1$ and $P(N)$ becomes the so called geometrical distribution. It should be stressed that already in [6] we have demonstrated that, indeed, observed single particle energy distributions, $f(E)$, follow Tsallis form with parameter $q$ coinciding with that obtained from the corresponding NB multiplicity distributions, $P(N)$. In this way parameter $q$ occurs as some unifying factor connecting two different experimental information, $f(E)$ and $P(N)$.

When dealing with heavy ion collisions one encounters situation where only a part of nucleus participates actively in the collision (usually it is given by the number of nucleons participating in the collision, $N_{P}$ ). The remain part acts as spectators. However, in such situation one can expect that there exists some energy transfer taking place between those two parts. As shown in $[1,5]$ this can be accounted for by replacing temperature $T$ in Eqs. (3) and (4) by some effective temperature, $T_{\text {eff }}=T_{0}+(q-1) T_{\text {visc }}$ where $T_{0}=\langle T\rangle$ and $T_{\text {visc }}$ is parameter describing details of such energy transfer [5]. As demonstrated in [1] the use of the notion of $T_{\text {eff }}$ allows to observe that $\operatorname{Var}(N) /\langle N\rangle$ 
and $\langle N\rangle$ are mutually connected, see Fig. 1a. If $T_{\text {eff }}$ depends on $q$, the dependence $\operatorname{Var}(N) /\langle N\rangle$ on the number of participants $N_{P}$ is connected to the dependence of $\langle N\rangle$ on $N_{P}$. Accounting for the fluctuations of accessible energy one gets that $q-1=\operatorname{Var}(T) /\langle T\rangle^{2}=(1-\xi) / C_{V}$ where $C_{V}$ is heat capacity and $\xi \simeq N_{P} / A$ is parameter depending on the size of the hadronizing source. As one can see in Fig. 1a this is experimentally observed dependence. The second example deals with the fact
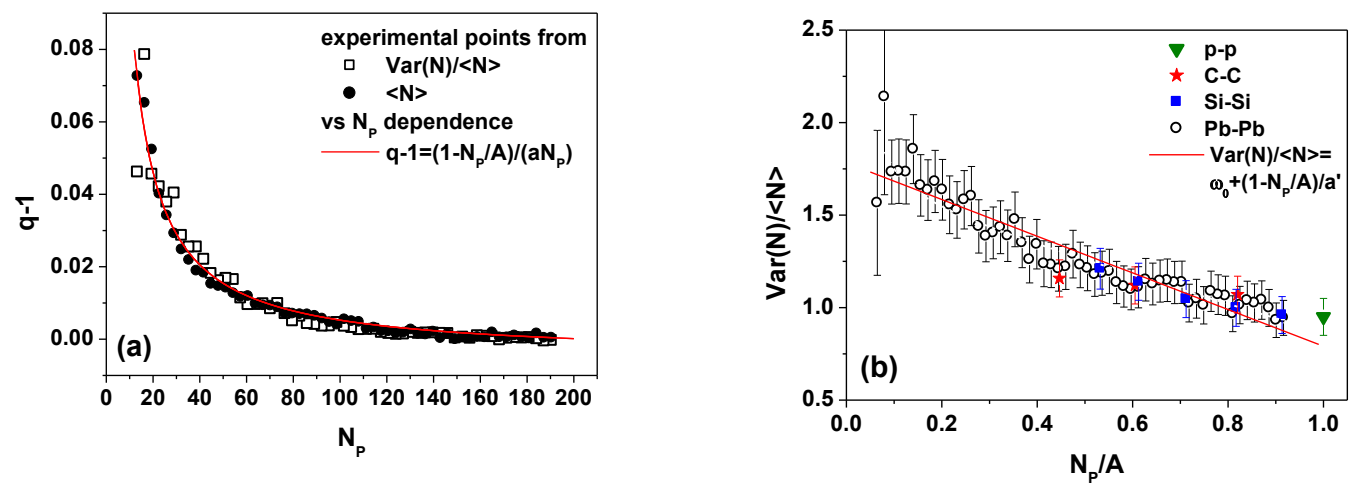

Figure 1: Examples of obtained results [1]. (a) Squares were obtained from the experimentally measured $\operatorname{Var}(N) /\langle N\rangle$ versus $N_{P}$ dependence whereas circles from the $\langle N\rangle$ versus $N_{P}$ dependence. The solid line shows our the dependence of $q-1$ versus $N_{P}$ obtained from our considerations [1]. (b) Scaled variance of the multiplicity distribution of negatively charged particles produced in $\mathrm{p}+\mathrm{p}$, semicentral $\mathrm{C}+\mathrm{C}$, semicentral $\mathrm{Si}+\mathrm{Si}$, and $\mathrm{Pb}+\mathrm{Pb}$ collisions as function of the fraction of nucleons participating in the collision, $N_{P} / A$. The experimental data of NA49 are compared with our predictions (see Fig. 4 of [1] for details in what concerns references and values of parameters).

that NA49 data (see [1] for details) show that $\operatorname{Var}(N) /\langle N\rangle$ changes rather strongly with $N_{P}$. For peripheral collisions (i.e., for small $N_{P}$ ) one observes marked deviation of $\operatorname{Var}(N) /\langle N\rangle$ from unity, see Fig. 1b. Notice that if $\langle N\rangle$ is linear in $N_{P}$ then $\operatorname{Var}(N) /\langle N\rangle$ is constant (this is also the case if one neglects the possible fluctuations of the full energy accessible for production of secondaries). Therefore the experimental fact that $\operatorname{Var}(N) /\langle N\rangle$ decreases with increasing $N_{P}$ indicates nonlinear dependence of $\langle N\rangle$ on the number of participants $N_{P}$.

We argue [1] that the observed system size dependence of $q$ is due to the finiteness of the hadronizing source ( $q=1$ corresponds to an infinite, thermalized source with a fixed temperature, whereas $q>1$ corresponds to a finite source in which both the temperature and energy fluctuate).

\section{References}

[1] G. Wilk and Z. Włodarczyk, Phys. Rev. C 79, 054903 (2009).

[2] G. Wilk, and Z. Włodarczyk, Phys. Rev. Lett. 84, 1770 (2000).

[3] T.S. Biró (editor), Topical Issue on Statistical Power Law Tsallis in High-Energy Phenomena, Eur. Phys. J. A 40 (3) (2009).

[4] G. Wilk and Z. Włodarczyk, Physica A 376, 279 (2007).

[5] G. Wilk and Z. Włodarczyk, Eur. Phys. J. A 40, 299 (2009).

[6] F.S. Navarra, O.V. Utyuzh, G. Wilk and Z. Włodarczyk, Phys. Rev. D67, 114002 (2003). 